\title{
Avaliação clínica e incidência de endof talmite infecciosa e não-infecciosa após injeção intravítrea de acetonida de triancinolona
}

\author{
Clinical characteristics and incidence of infectious and non-infectious \\ endoph thalmitis after in tra vitreous injection of triamcinolone acetonide
}

\author{
Érika Magalhães ${ }^{1}$ \\ Patrícia Nehemy ${ }^{2}$ \\ Daniel Vítor de Vasconcelos Santos ${ }^{3}$ \\ Gildo Fujji ${ }^{4}$ \\ Márcio Bittar Nehemy ${ }^{5}$
}

Instituto da Visão e Serviço de Retina e Vítreo do Hospital São Geraldo da Faculdade de Medicina da Universidade Federal de Minas Gerais - UFMG - Belo Horizonte (MG) - Brasil.

Doutora em Oftalmologia pela Faculdade de Medicina da Universidade Federal de Minas Gerais - UFMG Belo Horizonte (MG) - Brasil.

${ }^{2}$ Aluna do Curso de Graduação em Medicina pela UFMG - Belo Horizonte (MG) - Brasil. Bolsista de Iniciação Científica - CNPQ.

Pós-Graduando (nível Doutorado) em Oftalmologia pela UFMG - Belo Horizonte (MG) - Brasil.

${ }^{4}$ Doutor em Oftalmologia pela Universidade Federal de São Paulo - UNIFESP - São Paulo (SP) - Brasil. Professor Adjunto da UFMG - Belo Horizonte (MG) Brasil.

Endereço para correspondência: Rua dos Otoni, 881 - 13ำ andar - Belo Horizonte (MG) CEP 30150-270 E-mail: ivisao@ivisao.com

Recebido para publicação em 30.09.2005

Última versão recebida em 13.03.2006

Aprovação em 27.03.2006

Nota Editorial: Depois de concluída a análise do artigo sob sigilo editorial e com a anuência do Dr. Haroldo Vieira de Moraes Jr. sobre a divulgação de seu nome comø revisor, agradecemos sua participação neste processo.

\begin{tabular}{|l|}
\hline RESUMO \\
\hline Objetivo: Relatar a incidência de endoftalmite infecciosa enão-infecciosa \\
após injeção intravítrea de $4 \mathrm{mg}$ de triancinolona (Kenalog ${ }^{\circledR}-40 \mathrm{mg} / \mathrm{ml} ;$ \\
$0,1 \mathrm{ml}$ ) e avaliar aspectos clínicos relevantes para o diagnóstico diferencial \\
entre estas duas entidades. Desenho: Estudo prospectivo não-concorrente. \\
Métodos: Foram analisados os prontuários de 121 pacientes (154 injeções) \\
que, consecutivamente, foram submetidos à injeção intravítrea de trianci- \\
nolona para o tratamento de diversas doenças coriorretinianas. Todas as \\
injeções foram realizadas em centro cirúrgico em condições de assepsia e \\
anti-sepsia, comuns às cirurgias oftalmológicas. Resultados: Nenhum \\
olho apresentou endoftalmite infecciosa. Dois olhos (1,29\%/injeção e \\
1,65\%/paciente) apresentaram endoftalmite não-infecciosa caracterizada \\
pela observação, no primeiro dia pós-operatório, de baixa de acuidade \\
visual, hiperemia, hipópio e reação inflamatória no vítreo. Estes dois olhos \\
evoluíram com resolução do quadro inflamatório, após o uso de corticóide \\
tópico e subconjuntival. Conclusão: Na presente série, nenhum olho \\
apresentou endoftalmite infecciosa. A ocorrência de endoftalmite não- \\
infecciosa após a injeção intravítrea de triancinolona é relativamente rara \\
e, geralmente, pode ser diferenciada da endoftalmite infecciosa por meio \\
da análise criteriosa das suas manifestações clínicas.
\end{tabular}

Descritores: Coróide; Doenças retinianas; Endoftalmite; Infecções oculares; Injeções; Corpo vítreo; Triancinolona acetonida

\section{INTRODUÇ̃̃̃O}

A triancinolona é um corticosteróide potente, de depósito, que atua inibindo a síntese de prostaglandinas e leucotrienos, com consequiente efeito anti-inflamatório $^{(1)}$. Nos últimos anos, a triancinolona intravítrea vem sendo amplamente utilizada no tratamento de diversas doenças, tais como o edema macular associado à pseudofacia ${ }^{(2-4)}$, retinopatia diabética ${ }^{(5-7)}$, oclusão de veia central da retina ${ }^{(8)}$, vasculites retinianas ${ }^{(9)}$ e membrana neovascular sub-retiniana de várias etiologias ${ }^{(10-14)}$. A injeção de um corticóide de depósito, na cavidade vítrea, permite um efeito imediato, potente e de duração intermediária sobre a retina ${ }^{(15)}$. Ela pode, entretanto, levar a efeitos adversos significativos, tais como, catarata, hipertensão ocular, descolamento de retina e endoftalmite, que é uma das mais graves e temidas complicações desse procedimento ${ }^{(16-19)}$.

Deve-se ressaltar que o efeito imunossupressivo dos corticosteróides pode, eventualmente, tornar o olho mais vulnerável à infecção ou mascarar o processo infeccioso ${ }^{(15,19)}$. Endoftalmite não-infecciosa após injeção de 
triancinolona tem também sido relatada ${ }^{(15-19)}$. Nestes casos, há um verdadeiro processo inflamatório intra-ocular sem um componente infeccioso ${ }^{(15)}$. O conhecimento dessas duas entidades, bem como a sua diferenciação, é fundamental para permitir um tratamento oportuno e adequado.

O objetivo deste trabalho é relatar a incidência de endoftalmite infecciosa e não-infecciosa, após injeção intravítrea de triancinolona, em uma série de pacientes com doenças coriorretinianas, e avaliar aspectos clínicos relevantes para o diagnóstico diferencial entre estas duas entidades clínicas.

\section{MÉTODOS}

Foram analisados os prontuários de 121 pacientes submetidos, consecutivamente, à injeção intravítrea de triancinolona, no Instituto da Visão, no período de janeiro de 2001 a dezembro de 2003. Todos os pacientes receberam o tratamento em apenas um olho. Trinta e três olhos foram submetidos a mais de uma injeção, o que resultou em um total de 154 injeções intra-oculares.

A acetonida de triancinolona $\left(\operatorname{Kenalog}^{\circledR}\right)$ foi utilizada na dose de $4 \mathrm{mg}$ (40 mg/ml; 0,1 ml). A injeção foi realizada, em todos os pacientes, em centro cirúrgico, com técnica estritamente asséptica. Antes da injeção, instilou-se colírio de iodopovidine a 5\% no saco conjuntival. Após cinco minutos, os cílios eram isolados utilizando-se campo cirúrgico plástico e blefarostato, e o saco conjuntival era irrigado com solução salina balanceada. Após paracentese e aspiração de aproximadamente $0,1 \mathrm{ml}$ de humor aquoso da câmara anterior, procedeu-se à injeção de $0,1 \mathrm{ml}$ de triancinolona na cavidade vítrea, por via pars plana. Para tanto, utilizou-se uma agulha de 25 gauge que foi introduzida no quadrante temporal inferior a 3,5 mm (olhos afácicos ou pseudofácicos) ou $4 \mathrm{~mm}$ do limbo (olhos fácicos). Todos os pacientes fizeram uso de antibiótico tópico (ciprofloxacina 0,3\%), 4 vezes ao dia, durante 5 dias, a partir do primeiro dia pós-operatório. Não foi utilizado colírio de antibiótico no pré-operatório.

Todos os pacientes foram re-examinados no primeiro dia pós-operatório. Pacientes com hipópio e/ou reação inflamatória na câmara anterior ou no vítreo foram seguidos, inicialmente a cada seis horas e, diariamente após o segundo dia, até que houvesse melhora dos sinais clínicos de inflamação. Os pacientes eram, então, seguidos com exames regulares, em intervalos variáveis, de acordo com a doença coriorretiniana de base.

\section{RESULTADOS}

No primeiro dia de pós-operatório, dois olhos (1,29\%/injeção e 1,65\%/paciente) apresentaram quadro clínico caracterizado por baixa da acuidade visual, hiperemia bulbar (1+ a 2+), hipópio e reação inflamatória no vítreo $(2+$ a $3+$ de células). Nenhum dos pacientes relatou dor ocular (Figura 1). Os 152 olhos restantes não apresentaram reação inflamatória significativa.

A ausência de dor ocular, assim como, o aparecimento do quadro no primeiro dia de pós-operatório, não parecia sugerir

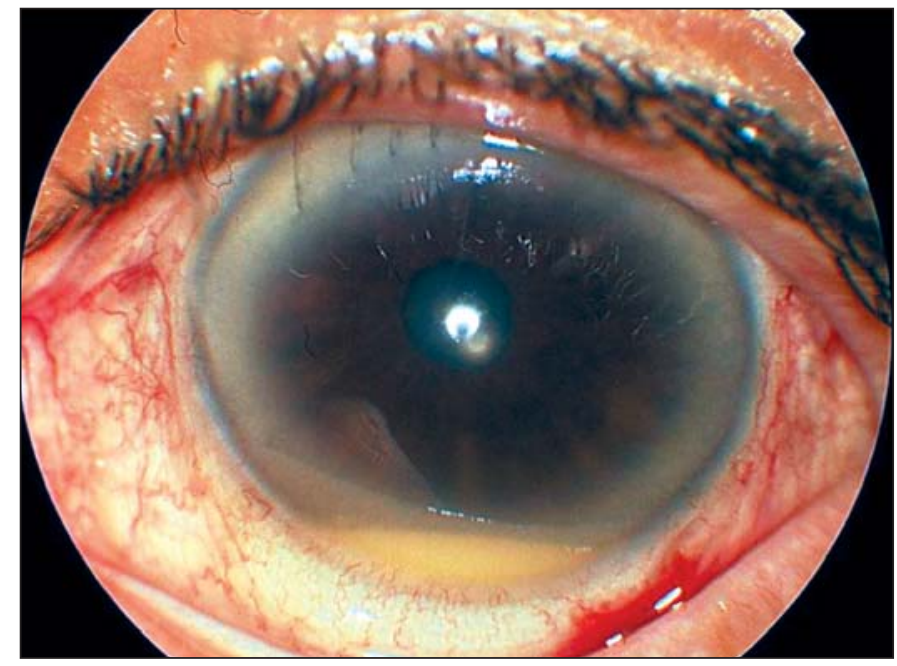

Figura 1 - Aspecto clínico 24 horas após injeção intravítrea de triancinolona. Observa-se hiperemia bulbar moderada e hipópio.

uma endoftalmite infecciosa. Por esta razão, optou-se por realizar uma injeção subconjuntival de corticóide (dexametasona - $1 \mathrm{mg}$ ) e seguir os pacientes com observação rigorosa. Os pacientes foram reavaliados 6, 12, 24, 48 e 72 horas após o procedimento. Ambos os olhos apresentaram sinais de melhora progressiva do quadro, nos dias seguintes (Figura 2). Em sua reavaliação, em três meses, nenhum dos dois olhos apresentou reação inflamatória residual.

\section{DISCUSSÃO}

Durante as duas últimas décadas, o uso de injeções intravítreas vem sendo utilizado de forma crescente no tratamento de diversas doenças intra-oculares, particularmente nas doenças do segmento posterior ${ }^{(15)}$. A injeção de drogas na cavidade vítrea tem sido realizada com a finalidade de se atingir uma concentração vítrea acima da obtida com a administração por via sistêmica, e, como meio de evitar eventuais efeitos sistêmicos $\operatorname{adversos}^{(15)}$.

Recentemente, a administração intravítrea da triancinolona vem sendo amplamente utilizada no tratamento de diversas doenças coriorretinianas, tais como o edema macular ${ }^{(3-10)}$ e a neovascularização coroideana ${ }^{(11-14)}$.

Os efeitos adversos mais freqüentemente relatados após a injeção intravítrea de triancinolona são a catarata e a hipertensão ocular ${ }^{(15-17)}$. A endoftalmite, embora mais rara, é potencialmente mais grave $\mathrm{e}^{(15-20)}$. A literatura caracteriza as endoftalmites com diversos termos, tais como "infecciosa", "não-infecciosa", "estéril", "pseudo-endoftalmite" e "endophthalmitislike". Infelizmente, alguns destes termos têm sido usados de forma inconsistente e, algumas vezes, intercambiáveis, causando dificuldades de interpretação $0^{(15)}$. A definição correta e a diferenciação entre estas entidades é fundamental para o diagnóstico correto e tratamento preciso e oportuno.

As endoftalmites infecciosas e não-infecciosas devem ser 

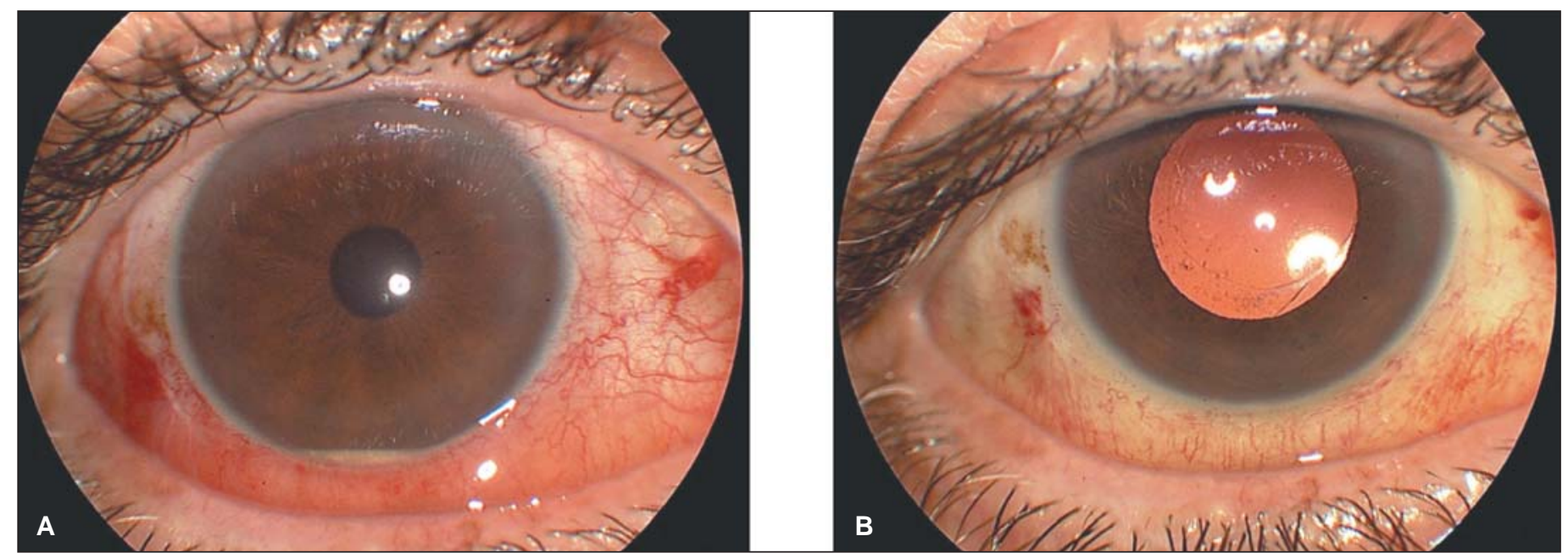

Figura 2 - A: aspecto clínico 24 horas após injeção intravítrea de triancinolona. Observa-se hiperemia moderada, quemose, pequena hemorragia subconjuntival temporalmente na conjuntiva bulbar e pequeno hipópio; B: aspecto clínico do mesmo olho da figura A, 72 horas após a injeção. Observa-se discreta hiperemia bulbar, redução do processo inflamatório e ausência de hipópio.

diferenciadas da pseudo-endoftalmite, que é relativamente comum, e deve-se à passagem das partículas da triancinolona para a câmara anterior, formando um pseudo-hipópio ${ }^{(16-20)}$. Esta diferenciação pode apresentar dificuldades. Mas, apesar disto, algumas características podem auxiliar no diagnóstico diferencial. O pseudo-hipópio é mais comum em olhos afácicos ou pseudofácicos, particularmente nos que apresentam descontinuidade da cápsula posterior. Em alguns desses casos, pode-se surpreender a passagem da droga para a câmara anterior no per-operatório ou nas primeiras horas após a injeção. Tipicamente, estes olhos apresentam-se sem baixa de visão significativa, hiperemia ou outros sinais de inflamação.

A endoftalmite infecciosa já foi relatada após injeção intravítrea de triancinolona ${ }^{(19)}$. Em trabalho de revisão recentemente publicado, relatou-se uma incidência global de endoftalmite com cultura positiva de $0,6 \%$ /injeção, e de $0,6 \%$ /paciente, após injeção intravítrea de triancinolona ${ }^{(15)}$. Na presente série de casos, observou-se ausência de casos de endoftalmite infecciosa. É possível que a ausência desta complicação seja devida, pelo menos em parte, ao fato de que todas as injeções terem sido efetuadas em centro cirúrgico, com uso de iodopovidine no saco conjuntival, isolamento dos cílios, e com cuidados de assepsia, próprios de um procedimento cirúrgico oftalmológico.

Alguns autores levantaram a hipótese de que casos de endoftalmite não-infecciosa possam ser causados por uma reação inflamatória a alguma substância na formulação da triancinolona ${ }^{(20)}$. Dessa maneira, o termo endoftalmite "não-infecciosa" ou "asséptica" deve ser reservado para estes casos, nos quais há um verdadeiro processo inflamatório intra-ocular secundário à injeção intravítrea de triancinolona e sem um componente infeccioso ${ }^{(15)}$. O Kenalog ${ }^{\circledR}$ injetável contém álcool benzil a $0,99 \%$, sódio carboximetilcelulose a $0,75 \%$ e polissorbato 80 a 0,04\%, em suspensão. Hidróxido de sódio ou ácido hidroclórico podem também estar presentes para ajustar o $\mathrm{pH}$ para 5,0 a 7,5. A presença desses elementos químicos pode servir como um estímulo potencial para uma reação inflamatória dentro do olho. É possível que o desenvolvimento de uma formulação de triancinolona que não apresente agentes conservantes, para injeção intravítrea, possa reduzir o risco de endoftalmite não-infeccio$\mathrm{sa}^{(15)}$. Toxinas bacterianas podem também estar presentes em soluções estéreis e causar reação inflamatória ${ }^{(20)}$.

É importante ressaltar que uma endoftalmite com cultura negativa não implica, necessariamente, em um processo nãoinfeccioso, já que, freqüentemente, a endoftalmite infecciosa após injeção intravítrea de triancinolona pode ter cultura negativa $^{(15)}$. No presente estudo, observou-se uma incidência de endoftalmite não-infecciosa de 1,29\%/injeção e 1,65\%/paciente. Estes percentuais são comparáveis com dados da literatura que relatam uma incidência de 1,6\%/injeção de endoftalmite não-infecciosa presumível ou pseudo-endoftalmite, após injeção de triancinolona intravítrea ${ }^{(20)}$. Na presente série, não foi possível identificar qualquer fator de risco para a ocorrência da endoftalmite não-infecciosa. A baixa incidência e o número relativamente pequeno de pacientes não permitiu uma análise conclusiva a esse respeito.

No presente estudo, algumas manifestações sugeriram não se tratar de endoftalmite infecciosa. Entre estas, destacam-se a apresentação precoce e a ausência de dor. Nesta série, ambos os casos apresentaram o quadro pseudo-infeccioso no primeiro dia após a injeção. Trabalho recentemente publicado mostrou que de oito casos de endoftalmite infecciosa após injeção intravítrea de triancinolona apenas um se apresentou no primeiro dia após a injeção ${ }^{(19)}$. Nenhum dos pacientes deste estudo apresentou dor, sintoma que ocorre na maioria das endoftalmites infecciosas agudas, particularmente, nas que se apresentam nas primeiras 24 horas $^{(21)}$. A diminuição da acuidade visual, que freqüentemente ocorre na vigência de endoftalmite infecciosa após a cirurgia da catarata, é de difícil valorização em séries como esta, em que a maioria dos pacientes já apresenta uma baixa da acuidade visual significativa antes da injeção ${ }^{(15)}$. Neste 
estudo, entretanto, ambos os olhos apresentaram baixa de acuidade visual adicional. Esta observação sugere que a baixa de visão não deve, portanto, ser usada como critério para o diagnóstico diferencial entre endoftalmite infecciosa e não-infecciosa. O fato de que ambos os olhos apresentaram evolução favorável, com o uso de corticóide tópico e subconjuntival, e sem tratamento específico para endoftalmite infecciosa, corroboram a hipótese de que se tratava de uma endoftalmite não-infecciosa. No presente estudo, optou-se por uma avaliação clínica cuidadosa e frequiente, antes de se considerar procedimentos mais invasivos, que incluiriam a punção da câmara anterior, da cavidade vítrea, injeção de antibióticos intra-oculares e, eventualmente, a vitrectomia.

\section{CONCLUSÃO}

Apesar do número relativamente pequeno de casos de endoftalmite não-infecciosa, a análise da sua evolução sugere que os critérios clínicos adotados permitiram um manejo adequado dos pacientes.

Concluindo, nenhum caso de endoftalmite infecciosa ocorreu nesta série de pacientes. A ocorrência de endoftalmite não-infecciosa após a injeção intravítrea de triancinolona é relativamente rara e, geralmente, pode ser diferenciada da endoftalmite infecciosa por meio da análise criteriosa das suas manifestações clínicas.

\section{ABSTRACT}

Purpose: To report the incidence of infectious and noninfectious endophthalmitis after intravitreal injection of $4 \mathrm{mg}$ of triamcinolone acetonide $\left(\mathrm{Kenalog}^{\circledR}-40 \mathrm{mg} / \mathrm{ml} ; 0.1 \mathrm{ml}\right)$ and to evaluate distinguishing characteristics that may assist the clinician in differentiating these entities. Design: Observational nonconcurrent prospective study. Methods: Charts of 121 patients (154 injections) who consecutively underwent intravitreal injection of triamcinolone acetonide to treat various chorioretinal diseases were evaluated. All procedures were performed in an operating room with careful antiseptic protocol. Results: Two eyes (1.29\%/injection and $1.65 \% /$ patient) presented a noninfectious endophthalmitis characterized by decreased vision, hyperemia, hypopyon and vitreous inflammatory reaction, on the first day after the injection. These eyes were treated with topical and subconjunctival corticosteroids with complete resolution of the inflammatory reaction. Conclusion: In the present case series, no case of infectious endophthalmitis occurred. Despite being relatively rare, noninfectious endophthalmitis can be associated with intravitreal injection of triamcinolone simulating an infectious endophthalmitis. In selected cases, the differential diagnosis can be made solely by clinical evaluation.

Keywords: Choroid; Retinal diseases; Endophthalmitis; Eye infections; Injections; Vitreous body; Triamcinolone acetonide

\section{REFERÊNCIAS}

1. Hood PP, Cotter TP, Costello JF, Sampson AP. Effect of intravenous corticosteroid on ex vivo leukotriene generation by blod leucocytes of normal and asthmatic patients. Thorax. 1999;54(12):1075-82.

2. Scott IU, Flynn HW Jr, Rosenfeld PJ. Intravitreal triamcinolone acetonide for idiopathic cystoid macular edema. Am J Ophthalmol. 2003;136(4):737-9.

3. Jonas JB, Kreissig I, Degenring RF. Intravitreal triamcinolone acetonide for pseudophakic cystoid macular edema. Am J Ophthalmol. 2003;136(2):384-6. Comment in: Am J Ophthalmol. 2003;136(6):575-6; author reply 1192-3.

4. Benhamou N, Massin P, Haouchine B, Audren F, Tadayoni R, Gaudric A. Intravitreal triamcinolone for refractory pseudophakic macular edema. Am J Ophthalmol. 2003;135(2):246-9.

5. Jonas JB, Kreissig I, Sofker A, Degenring RF. Intravitreal injection of triamcinolone for diffuse diabetic macular edema. Arch Ophthalmol. 2003;121(1): 57-61. Comment in: Arch Ophthalmol. 2004;122(7):1082-3; author reply 1086-8. Arch Ophthalmol. 2004;122(7):1082; author reply 1086-8. Arch Ophthalmol. 2004;122(7):1083-5; author reply 1086-8. Arch Ophthalmol. 2004; 122(7):1083; author reply 1086-8. Arch Ophthalmol. 2004;122(7):1085-6; author reply 1086-8.

6. Martidis A, Duker JS, Greenberg PB, Rogers AH, Puliafito CA, Reichel E, Baumal C. Intravitreal triamcinolone for refractory diabetic macular edema. Ophthalmology. 2002;109(5):920-7.

7. Jonas JB, Sofker A. Intraocular injection of crystalline cortisone as adjunctive treatment of diabetic macular edema. Am J Ophthalmol. 2001;132(3):425-7.

8. Greenberg PB, Martidis A, Rogers AH, Duker JS, Reichel E. Intravitreal triamcinolone acetonide for macular oedema due to central retinal vein occlusion. Br J Ophthalmol. 2002;86(2):247-8

9. Godoy G, Finamor LP, Andrade R, Muccioli C. Triancinolona intravítrea em vasculite retiniana - relato de caso. Arq Bras Oftalmol. 2004;67(6):953-6.

10. Rechtman E, Allen VD, Danis RP, Pratt LM, Harris A, Speicher MA. Intravitreal triamcinolone for choroidal neovascularization in ocular histoplasmosis syndrome. Am J Ophthalmol. 2003;136(4):739-41.

11. Jonas JB, Akkoyun I, Budde WM, Kreissig I, Degenring RF. Intravitreal reinjection of triamcinolone for exudative age-related macular degeneration. Arch Ophthalmol. 2004;122(2):218-22.

12. Gillies MC, Simpson JM, Luo W, Penfold P, Hunyor AB, Chua W, et al. A randomized clinical trial of a single dose of intravitreal triamcinolone acetonide for neovascular age-related macular degeneration: one-year results. Arch Ophthalmol. 2003;121(5):667-73. Comment in: Arch Ophthalmol. 2004;122(10): 1571-2; author reply 1572 .

13. Spaide RF, Sorenson J, Maranan 1. Combined photodynamic therapy with verteporfin and intravitreal triamcinolone acetonide for choroidal neovascularization. Ophthalmology. 2003;110(8):1517-25.

14. Jonas JB, Kreissig I, Hugger P, Sauder G, Panda-Jonas S, Degenring R. Intravitreal triamcinolone acetonide for exudative age related macular degeneration. Br J Ophthalmol. 2003;87(4):462-8.

15. Jager RD, Aiello LP, Patel SC, Cunningham ET Jr. Risks of intravitreous injection: a comprehensive review. Retina, 2004;24(5):676-98.

16. Roth DB, Chieh J, Spirn MJ, Green SN, Yarian DL, Chaudhry N. Noninfectious endophthalmitis associated with intravitreal triamcinolone injection. Arch Ophthalmol. 2003;121(9):1279-82. Comment in: Arch Ophthalmol. 2004;122(11):1733; author reply 1733.

17. Sutter FK, Gillies MC. Pseudo-endophthalmitis after intravitreal injection of triamcinolone. Br J Ophthalmol. 2003;87(8):972-4.

18. Wingate RJ, Beaumont PE. Intravitreal triamcinolone and elevated intraocular pressure. Aust N Z J Ophthalmol. 1999;27(6);431-2.

19. Moshfeghi DM, Kaiser PK, Scott IU, Sears JE, Benz M, Sinesterra JP, et al. Acute endophthalmitis following intravitreal triamcinolone acetonide injection. Am J Ophthalmol. 2003;136(5):791-6. Comment in: Am J Ophthalmol. 2003; 136(5):918-9. Am J Ophthalmol. 2004;137(6):1158-9; author reply 1160-1. Am J Ophthalmol. 2004;137(6):1159-60; author reply 1160-1. Am J Ophthalmol. 2004;137(6):1166; author reply 1167 .

20. Nelson ML, Tennant MT, Sivalingam A, Regillo CD, Belmont JB, Martidis A. Infectious and presumed noninfectious endophthalmitis after intravitreal triamcinolone acetonide injection. Retina. 2003;23(5):686-9

21. Johnson MW, Doft BH, Kelsey SF, Barza M, Wilson LA, Barr CC, Wisniewski SR. The Endophthalmitis Vitrectomy Study Group. The Endophthalmitis Vitrectomy Study Group: Relationship between clinical presentation and microbiological spectrum. Ophthalmology, 1997;104(2):261-72. 